University of Warwick institutional repository: http://go.warwick.ac.uk/wrap This paper is made available online in accordance with publisher policies. Please scroll down to view the document itself. Please refer to the repository record for this item and our policy information available from the repository home page for further information.

To see the final version of this paper please visit the publisher's website. Access to the published version may require a subscription.

Author(s): G. R. Bell, T. S. Jones, and C. F. McConville Article Title: Accumulation layer profiles at InAs polar surfaces Year of publication: 1997

Link to published version: http://dx.doi.org/10.1063/1.120482

Publisher statement: none 


\title{
Accumulation layer profiles at InAs polar surfaces
}

\author{
G. R. Bell ${ }^{\mathrm{a})}$ \\ Department of Physics, University of Warwick, Coventry CV4 7AL, United Kingdom \\ T. S. Jones \\ Department of Chemistry, Imperial College, London SW7 2AY, United Kingdom \\ C. F. McConville \\ Department of Physics, University of Warwick, Coventry CV4 7AL, United Kingdom
}

(Received 5 August 1997; accepted for publication 21 October 1997)

\begin{abstract}
High resolution electron energy loss spectroscopy, dielectric theory simulations, and charge profile calculations have been used to study the accumulation layer and surface plasmon excitations at the In-terminated (001)- $(4 \times 1)$ and $(111) A-(2 \times 2)$ surfaces of InAs. For the (001) surface, the surface state density is $4.0 \pm 2.0 \times 10^{11} \mathrm{~cm}^{-2}$, while for the (111)A surface it is $7.5 \pm 2.0 \times 10^{11} \mathrm{~cm}^{-2}$, these values being independent of the surface preparation procedure, bulk doping level, and substrate temperature. Changes of the bulk Fermi level with temperature and bulk doping level do, however, alter the position of the surface Fermi level. Ion bombardment and annealing of the surface affect the accumulation layer only through changes in the effective bulk doping level and the bulk momentum scattering rate, with no discernible changes in the surface charge density. (C) 1997 American Institute of Physics. [S0003-6951(97)02751-4]
\end{abstract}

The polar surfaces of InAs have attracted significant interest in recent years due to the unusual position of the surface Fermi level, which lies above the conduction band minimum (CBM) for many surfaces. ${ }^{1-5}$ Fermi level stabilization above the CBM is allowed in this material because the $\Gamma$ point CBM is lower than the "charge neutrality level" or "effective midgap energy," at which the surface state energies are likely to lie. ${ }^{4-6}$ The specific surface states which act as donors are, however, unknown although a clear connection between the surface reconstruction and surface charge density has been established. ${ }^{1,4}$ Photoemission spectroscopy (PES) has been employed recently to reveal the presence of a high conduction band electron density near the (001) and (111) surfaces of InAs. ${ }^{4}$ High resolution electron energy loss spectroscopy (HREELS) can also be used to probe the surface accumulation layer by examining surface plasmon excitations. ${ }^{1-3}$ In this letter, we examine the effects of substrate temperature and the surface preparation procedure on the accumulation layer characteristics of polar InAs surfaces, using HREELS measurements combined with dielectric theory simulations ${ }^{7}$ and charge profile calculations. ${ }^{8,9}$

The experiments were performed in an ultrahigh vacuum system equipped with HREELS, low energy electron diffraction (LEED), an argon ion gun, and an atomic hydrogen source. The samples could be heated radiatively (300-600 K) while recording HREEL spectra, and moderately doped InAs(111)A substrates $\left(n \sim 1 \times 10^{17} \mathrm{~cm}^{-3}\right)$ and heavily doped InAs(001) substrates $\left(n \sim 5 \times 10^{18} \mathrm{~cm}^{-3}\right)$ were studied. Clean and ordered surfaces were prepared by one of three methods: cycles of argon ion bombardment and annealing (IBA); atomic hydrogen cleaning (AHC) at $670 \mathrm{~K}$ with a $\mathrm{H}^{*}$ exposure of $\sim 10 \mathrm{~kL}$; or thermal decapping (TDC) of an amorphous As capping layer. After sample preparation by

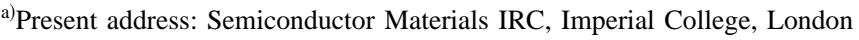
SW7 2BZ, UK.

${ }^{b)}$ Corresponding author. Electronic mail: C.F.McConville@warwick.ac.uk
}

any of these methods, a clear $(2 \times 2)$ LEED pattern was observed in the case of the (111) $A$ samples, and a "streaky $(4 \times 1)$ " pattern was seen in the case of the (001) samples. The latter pattern indicates one-dimensional disorder in the [110] (dimer bond) direction. This disorder was reduced on samples prepared by low energy IBA, especially when additional cycles were employed, as was indicated by an increased modulation of the intensity along the half-order streak. Specular HREEL spectra were recorded with electron incidence energies in the range 4-100 eV and an energy broadening of 6-10 meV full width at half maximum in the elastic peak.

HREEL spectra obtained from InAs(111)A-(2×2) surfaces prepared by AHC, with electron energies of 5 and 20 $\mathrm{eV}$ and sample temperatures of 300 and $550 \mathrm{~K}$, are shown in Fig. 1. The Fuchs-Kliewer phonon is observable as a shoul-

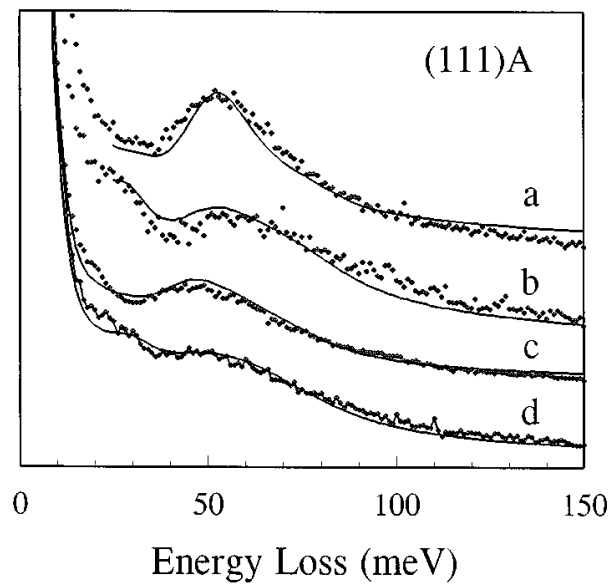

FIG. 1. Experimental HREEL spectra (dotted lines) and four-layer dielectric theory simulations (solid lines) for $\operatorname{InAs(111)A}$ samples $(n \sim 1$ $\times 10^{17} \mathrm{~cm}^{-3}$ ) prepared using AHC. Spectrum (a) was obtained with an electron energy of $20 \mathrm{eV}$ at a temperature of $550 \mathrm{~K}$, (b) at $5 \mathrm{eV}$ and $550 \mathrm{~K}$, (c) at $20 \mathrm{eV}$ and $300 \mathrm{~K}$, and (d) at $5 \mathrm{eV}$ and $300 \mathrm{~K}$. 


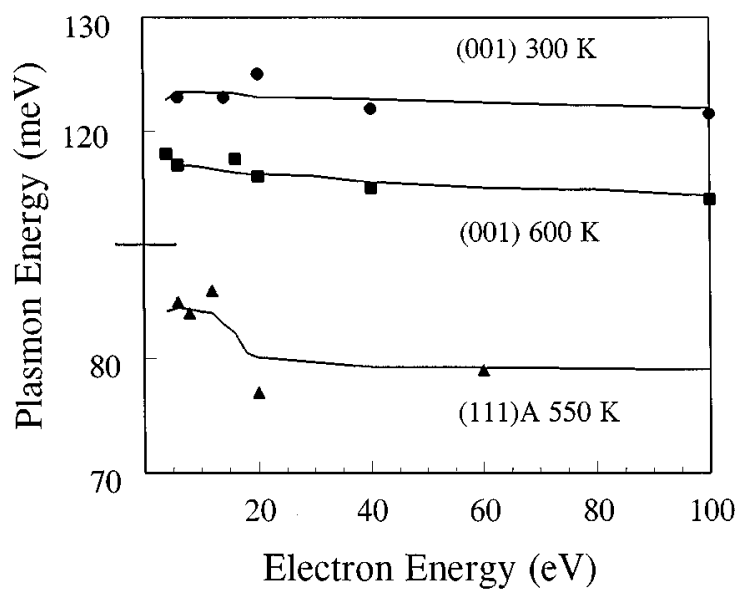

FIG. 2. The plasmon dispersion derived from experimental HREEL spectra recorded for $\operatorname{InAs}(001)\left(n \sim 5 \times 10^{18} \mathrm{~cm}^{-3}\right)$ and $\operatorname{InAs}(111) A \quad(n \sim 1$ $\times 10^{17} \mathrm{~cm}^{-3}$ ) samples prepared by IBA. For the (001) surfaces, the sample temperatures shown are $300 \mathrm{~K}$ (circles) and $600 \mathrm{~K}$ (squares), while for the (111) $A$ samples (triangles) the temperature is $550 \mathrm{~K}$. The solid lines are fits to the data based on three-layer dielectric theory simulations.

der on the loss side of the elastic peak $(\sim 24 \mathrm{meV})$, while the broad peak at around $55 \mathrm{meV}$ in each spectrum is due to the surface plasmon excitation. This peak is more pronounced at $550 \mathrm{~K}$ than at $300 \mathrm{~K}$, and lies at a slightly higher loss energy (54 meV compared with $48 \mathrm{meV}$ for the $20 \mathrm{eV}$ spectra). At both temperatures the plasmon shows some downward dispersion with increasing electron energy. The plasmon dispersion is shown in Fig. 2 for samples prepared by IBA rather than by AHC or TDC. The dispersion is most pronounced for the undoped (111)A samples, while the heavily doped (001) samples show only a slight downward dispersion with increasing electron energy, due to reduced spatial dispersion and band bending. The more dramatic dispersion evident in the case of the (111)A samples indicates the presence of a higher surface charge than on the (001) samples.

These data were analyzed using the semi-classical dielectric theory of HREELS to simulate complete HREEL spectra. $^{7}$ For each set of experimental spectra, a three-or four-layer model was used to produce simulated spectra across the entire electron energy range. The plasma dielectric function was based on the hydrodynamic model, with the plasmon lifetimes, spatial dispersion coefficients, and plasma frequencies as parameters. ${ }^{2}$ Good fits were obtained with only the plasmon lifetimes in each active layer being adjusted as a function of electron energy, which is required to incorporate the separate damping mechanisms for the plasmon excitations. ${ }^{2,8}$ The layer thicknesses and plasma parameters (apart from the lifetimes) were estimated using the modified Thomas-Fermi approximation (MTFA) ${ }^{9,10}$ with a temperature-dependent bulk band gap. The MTFA accounts for both the nonparabolicity of the conduction band and the potential barrier of the surface to the electron wave functions. ${ }^{11}$ These are both very important effects, since the surface potential barrier results in the formation of a plasmon-free surface "dead layer" to which the low energy HREELS response is highly sensitive, ${ }^{1-3,12}$ and the Fermi level lies far above the CBM in the accumulation layer.

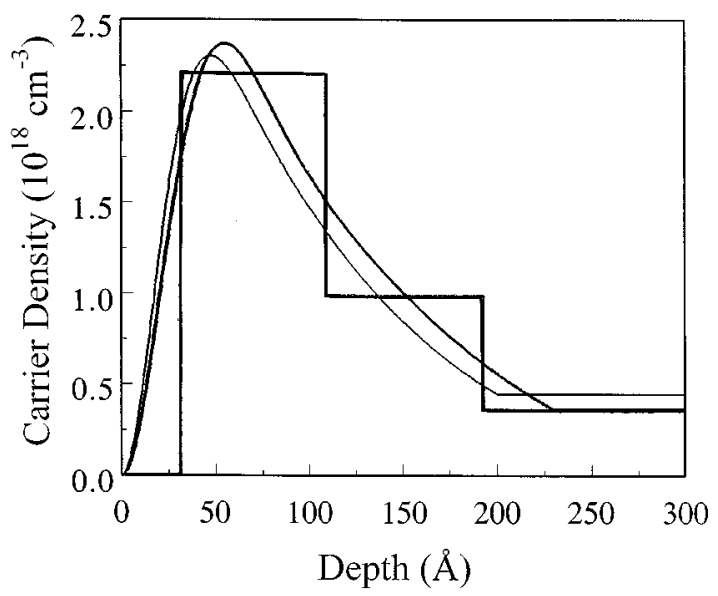

FIG. 3. The MTFA electron density profile for InAs(111)A-(2×2) surfaces $\left(n \sim 1 \times 10^{17} \mathrm{~cm}^{-3}\right)$ prepared by AHC at temperatures of $300 \mathrm{~K}$ (heavy solid curve) and $550 \mathrm{~K}$ (light solid curve). Also shown is the four-layer profile used to simulate the experimental HREEL spectra obtained at $300 \mathrm{~K}$.

Using the MTFA, both the local plasma frequency and the spatial dispersion coefficient were calculated as a function of depth. The calculated plasma frequency profiles were split up into layered models for HREELS calculations, with the uppermost layer having a zero plasma frequency and the lowest layer being semi-infinite. To simplify this process, triangular potential wells were used for the MTFA calculations. Deviations between plasma frequency profiles based on triangular wells and full classical potentials were not significant in terms of the HREELS simulations. The parameters of the MTFA calculation in this scheme are the temperature, the potential gradient (which determines the surface state density), and the surface and bulk Fermi levels. The bulk Fermi level and temperature determine the plasma frequency at large depths $(>200 \AA)$, and the plasmon frequency at the higher electron energies employed is most sensitive to this value (effective probing depth $>500 \AA$ ). The surface Fermi level and the width of the potential well determine the accumulation layer shape, which governs the plasmon behavior at lower electron energies. However, the HREELS response is not particularly sensitive to the fine details of the accumulation layer shape due to the "smearing" of the depth-resolved information over the effective probing depth. ${ }^{12}$ This justifies the approach of using layered models to represent the smooth plasma frequency profile, but sets a limit on the accuracy to which the accumulation layer characteristics can be obtained. This is reflected in the error values for the surface state densities and the surface Fermi levels.

Calculated charge profiles for $\operatorname{InAs}(111) A$ samples $\left(n \sim 1 \times 10^{17} \mathrm{~cm}^{-3}\right)$ prepared by AHC are given in Fig. 3 for temperatures of 300 and $550 \mathrm{~K}$. A histogram approximation, representing the four-layer model used for the HREELS simulations, is also shown for the $300 \mathrm{~K}$ curve. For clarity, the corresponding $550 \mathrm{~K}$ histogram is not shown (similar profiles and histograms were generated for each HREELS data set). The bulk carrier concentration is slightly higher in the case of the $550 \mathrm{~K}$ spectrum, due to an increased intrinsic contribution. This increases the bulk plasma frequency by 6 
$\mathrm{meV}$, which accounts for the increase of the observed plasmon frequency in the highest energy HREEL spectra from $47(300 \mathrm{~K})$ to $54 \mathrm{meV}(550 \mathrm{~K})$. Note that for heavily doped InAs and InSb, the plasma frequency drops with increasing temperature while the carrier concentration is nearly independent of temperature. ${ }^{2,13}$ This is because of the increased electron effective mass at the Fermi level and the negligible intrinsic contribution. The effect is seen clearly in Fig. 2, where the observed plasmon frequencies drop by around 6 $\mathrm{meV}$ for the heavily doped IBA-treated (001) sample. MTFA calculations for the heavily doped samples (prepared by both IBA and TDC) also indicated a temperature-independent bulk carrier concentration. The most important point is that for doping levels across the range $10^{17}-10^{19} \mathrm{~cm}^{-3}$, these temperature-induced changes in the surface plasmon behaviour are a consequence of bulk band structure considerations alone. The surface Fermi level for the (111)A substrates prepared by AHC was found to be $210 \mathrm{meV}$ at $300 \mathrm{~K}$, dropping to $180 \mathrm{meV}$ at $550 \mathrm{~K}$, while the band bending was independent of temperature.

After IBA, the effective bulk carrier concentration of all the samples was raised, with the increase being greater for higher ion energies and for incidence angles closer to the surface normal. ${ }^{14}$ For example, the plasma frequency in the (111)A samples at $550 \mathrm{~K}$ was increased from $\sim 55 \mathrm{meV}$ [Fig. 1 curves (a) and (b)] to $\sim 80 \mathrm{meV}$ (Fig. 2, lowest curve) by using IBA with $400 \mathrm{eV}$ ions incident at $45^{\circ}$ to the surface normal. It was also found that the plasmon lifetime was reduced, especially in the semi-infinite layer. ${ }^{8}$ These effects are both due to the thick ( $>500 \AA$ ) damage-diffused region present after IBA. ${ }^{14}$ However, no significant change was found in the surface charge density (although the surface and bulk Fermi levels were both raised). This implies that the IBA process does not change the surface density of donorlike defects, despite dramatically increasing their bulk density in the subsurface region of the material. Since the onedimensional disorder seen in the LEED patterns was reduced by the IBA process on the (001) surfaces, it is clear that defects associated specifically with this disorder are not donor-like. This is consistent with recent studies employing scanning tunneling microscopy and spectroscopy. ${ }^{15}$ In fact, the value for the surface charge density derived for all of the In-terminated (001) surfaces here $\left(4 \times 10^{11} \mathrm{~cm}^{-2}\right)$ is in good agreement with the estimate of $\sim 5 \times 10^{11} \mathrm{~cm}^{-2}$ obtained from the HREELS studies of Noguchi et al. ${ }^{1}$ for $\operatorname{InAs}(001)-(4 \times 2)$ surfaces. Their low-doped samples were grown in situ by molecular beam epitaxy (MBE), and showed no one-dimensional disorder. The low doping level $\left(\sim 1 \times 10^{16} \mathrm{~cm}^{-3}\right)$ indicates that doping itself has no effect on the surface charge density through surface defect generation.

In contrast, for the (111) $A$ samples, the surface state density was $(7.5 \pm 2.0) \times 10^{11} \mathrm{~cm}^{-3}$, again independent of temperature and surface preparation method. This value is approximately twice as large as the value for the Interminated (001) surface, and represents a density of about $0.1 \%$ of a monolayer. We note that the PES measurements of Olsson et al. ${ }^{4}$ indicate a larger band bending at Asterminated $(111) B-(2 \times 2)$ surfaces than at As-terminated (001)- $(2 \times 4)$ surfaces. It appears therefore, that the higher surface charge density at (111) surfaces is a general feature of both As- and In-terminated surfaces. This is very likely to have a similar origin to the contrast between $(001)-(4 \times 2)$ and $(001)-(2 \times 4)$ InAs surfaces prepared by MBE, which have been studied both by HREELS ${ }^{1}$ and more recently by scanning tunneling spectroscopy. ${ }^{16}$

Olsson $\mathrm{et} \mathrm{al}^{4}{ }^{4}$ found that the substrate doping level did not have a significant effect on the surface Fermi level, for either $n$-type $\left(\sim 5 \times 10^{16} \mathrm{~cm}^{-3}\right)$ or $p$-type $\left(2 \times 10^{17} \mathrm{~cm}^{-3}\right)$ samples. We find that the surface Fermi level does vary as a consequence of shifting the bulk Fermi level, either by changing the temperature or by changing the effective bulk doping level through IBA. However, the observed changes preserve the band bending (i.e., the surface and bulk Fermi levels undergo nearly identical shifts). In heavily doped samples, the drop of surface and bulk Fermi levels is around $60 \mathrm{meV}$ in the temperature range $300-600 \mathrm{~K}$, while it is around $30 \mathrm{meV}$ for the moderately doped (111)A samples. Detection of such small shifts is difficult with PES, but they have a measurable effect on the surface plasmon dispersion observed in HREELS.

In conclusion, we have investigated the accumulation layer at In-terminated InAs polar surfaces and its dependence on substrate temperature, bulk doping level, and method of surface preparation. The surface charge density and band bending appear to be independent of all these parameters, although changes of the bulk Fermi level due to changes in both the bulk doping level and temperature do affect the surface Fermi level. For the $(001)-(4 \times 1)$ surface, the surface charge density is $(4.0 \pm 2.0) \times 10^{11} \mathrm{~cm}^{-2}$, while for the (111) $A-(2 \times 2)$ surface it is $(7.5 \pm 2.0) \times 10^{11} \mathrm{~cm}^{-2}$. This difference is related to the different density of donor-like surface defects, in a manner analagous to the variation in surface charge between As- and In-terminated $\operatorname{InAs}(001)$ surfaces.

${ }^{1}$ M. Noguchi, K. Hirakawa, and I. Ikoma, Phys. Rev. Lett. 66, 2243 (1991).

${ }^{2}$ G. R. Bell, C. F. McConville, and T. S. Jones, Phys. Rev. B 54, 2654 (1996).

${ }^{3}$ Y. Chen, J. C. Hermanson, and G. J. Lapeyre, Phys. Rev. B 39, 12682 (1989).

${ }^{4}$ L. Ö. Olsson, C. B. M. Andersson, M. C. Håkansson, J. Kanski, L. Ilver, and U. O. Karlsson, Phys. Rev. Lett. 76, 3626 (1996).

${ }^{5}$ J. M. Woodall, J. L. Freeouf, G. D. Petit, T. Jackson, and P. Kirchner, J. Vac. Sci. Technol. 19, 626 (1981).

${ }^{6}$ J. Tersoff, Phys. Rev. Lett. 52, 465 (1984).

${ }^{7} \mathrm{Ph}$. Lambin, J. P. Vigneron, and A. A. Lucas, Comput. Phys. Commun. 60, 351 (1990).

${ }^{8}$ G. R. Bell, T. S. Jones, and C. F. McConville, Surface Sci. (accepted for publication).

${ }^{9}$ J. P. Zollner, T. Fielder, and G. Bobsch, Phys. Status Solidi B 134, 837 (1986).

${ }^{10}$ G. R. Bell, C. F. McConville, C. P. A. Mulcahy, and T. S. Jones, J. Phys.: Condens. Matter 9, 2903 (1997).

${ }^{11}$ H. Ubensee and G. Paasch, Phys. Rev. B 39, 1955 (1988).

${ }^{12} \mathrm{H}$. Ibach and D. L. Mills, Electron Energy Loss Spectroscopy and Surface Vibrations (Academic, New York, 1982).

${ }^{13}$ T. S. Jones, M. O. Schweitzer, N. V. Richardson, G. R. Bell, and C. F. McConville, Phys. Rev. B 51, 17675 (1995).

${ }^{14}$ G. R. Bell, C. F. McConville, and T. S. Jones, Appl. Surf. Sci. 104/105, 15 (1996).

${ }^{15}$ C. Kendrick, G. LeLay, and A. Kahn, Phys. Rev. B 54, 17877 (1996).

${ }^{16} \mathrm{H}$. Yamaguchi, J. G. Belk, J. L. Sudijono, T. S. Jones, and B. A. Joyce, Appl. Phys. Lett. (submitted). 